\title{
The PARF Antagonist AChE Inhibitor PMS777 Attenuates LPS-Induced Acute Neuroinflammation
}

\author{
Jinjia Hu, Juan Li, Zhang Wei, Hongyu Gu, Biyun Shao, Dingwen Long, Hongzhuan Chen*
}

Department of Anatomy and Pharmacology, Shanghai Jiaotong University, Shanghai, China.

E-mail: jjhu2609@126.com

Received October $9^{\text {th }}, 2011$; revised November $10^{\text {th }}, 2011$; accepted November $20^{\text {th }}, 2011$

\begin{abstract}
When injected into the fourth ventricle, the proinflammagen lipopolysaccharide (LPS) induces acute neuroinflammation in the whole brain of rats. The new compound PMS777 is a novel platelet-activating factor receptor (PAFR) antagonist and acetylcholinesterase (AChE) inhibitor. The current study determined whether PMS777 could provide neuroprotection from the cytotoxic effects associated with LPS-induced neuroinflammation. Acute LPS infusions impaired recognition in rats as measured by the Morris water maze. In addition, LPS infusions decreased the number of AChE positive cells, and increased the number of OX-42 immunoreactive microglia and GFAP immunoreactive astrocytes in the hippocampus, the cortex and the basal nuclei. Furthermore, acute infusions of LPS also impaired organelles associated with protein synthesis. Peripheral administration of PMS777 (i.e., intraperitoneal injection) protected against the impairment in recognition, and attenuated the cytotoxic effects of the acute inflammatory processes upon cholinergic cells, microglia, astrocytes and ultrastructure of hippocampal cells. Here, we propose that the cytotoxic effects of acute neuroinflammation may involve the release of PAF and loss of cholinergic neurons, and this mechanism leads to neuronal dysfunction and spatial memory impairment. The PAFR antagonist inhibitor and AChE inhibitor PMS777 could provide neuroprotection from the cytotoxic effects induced by LPS.
\end{abstract}

Keywords: Neuroinflammation, Spatial Memory, Acetylcholinesterase, Platelet-Activating Factor, Ultrastrurctural Analysis

\section{Introduction}

There is a consensus that the neuropathogenesis of HIV-1-associated dementia (HAD) is initiated by the production of infected and antigenically activated brainresident macrophages and microglia [1]. For instance, the extent of neurologic dysfunction (i.e., dementia) has been correlated with the total number of macrophages and activated microglia in the brain parenchyma, rather than the number of HIV-1-infected macrophages and microglia. Because the direct infection of neurons with HIV-1 is unlikely, these findings suggest that neuronal dysfunction and death are likely mediated by soluble proinflammatory cytokines that are released by macrophages and microglia. These cytokines can include tumor necrosis factor- $\alpha$ (TNF- $\alpha)$ and the phospholipid mediator platelet-activating factor (PAF) [1,2]. The loss of cholinergic neurons caused by proinflammatory mediator-related inflammation is also considered as the most important pathophysiological processes associated with $\mathrm{AD}$ brain [3]. Similar to AD, these proinflammatory processes in HAD may also induce the loss of cholinergic neurons. Therefore, inhibiting these inflammatory responses and increasing acetylcholine (ACh) concentration in brain by modulating acetylcholinesterase (AChE) activity has become important strategies for AD or HAD therapy.

Proinflammagen lipopolysaccharides (LPS) are a component of the gram-negative bacteria cell wall, and LPS has been used experimentally to stimulate the production of endogenous cytokines and complement proteins [4,5]. Following an injection of LPS into the brain, activated glia are the main source of the interleukins (IL), tumor necrosis factor- $\alpha$ (TNF- $\alpha$ ), PAF and prostanoids [6]. Several lines of evidence suggest that activated microglia and elevated cytokine levels are also associated with neuropathogenesis and neurodegenerative disorders, such 
as HAD [1]. In this context, we investigated the effects of PMS777, a PAFR antagonist and AChE inhibitor, in order to characterize its potential neuroprotective effects following LPS-induced cytotoxicity in the brains of young rats.

\section{Experimental Procedures}

\subsection{Animal Preparation}

Sixty Sprague-Dawley rats, approximately 5 months old and weighing $250 \mathrm{~g}$, were divided into 6 groups as following: control group, model (LPS) group, P0.25 group, P0.5 group, P1 group and BN group. Rats were anesthetized with $20 \%$ urethane $(1300 \mathrm{mg} / \mathrm{kg}$, i.p), and placed in a stereotaxic instrument. The incisor bar was set $3 \mathrm{~mm}$ below the earbars (i.e., plat skull) and the scalp was incised and retracted. A hole was drilled in appropriate location in the skull with a dental drill. LPS $(10 \mu \mathrm{g} / 100 \mathrm{~g}$, dissolved in artificial CSF, $5 \mu \mathrm{g} / \mu \mathrm{l}$ ) was injected into the 4th ventricle. Eight control rats were injected with only artificial CSF. The P0.25 group and P0.5 group and P1 group rats were treated with PMS777 one time a day (PMS777 dissolved in $0.01 \mathrm{M}$ PBS, $0.25 \mathrm{mg} / \mathrm{kg}$ or 0.5 $\mathrm{mg} / \mathrm{kg}$ and $1 \mathrm{mg} / \mathrm{kg}$, i.p.). BN group rats were treated with BN52021 one time a day (BN52021 dissolved in BMSO and then diluted in $0.01 \mathrm{M} \mathrm{PBS}, 5 \mathrm{mg} / \mathrm{kg}$, i.p.).

\subsection{Behavioral Testing}

Behavioral testing [7] began three days after injection. Rats were introduced into the perimeter of a circular water-filled tank $120 \mathrm{~cm}$ in diameter and $30 \mathrm{~cm}$ in height, with a video camera suspended $170 \mathrm{~cm}$ above the liquid surface. Tracking software (DigBehv-MM, Jiliang Soft Tech Co.) recorded the path of the rodent by light/dark contrast detection for subsequent analysis. A square "escape platform" $(9 \mathrm{~cm} \times 9 \mathrm{~cm})$ was placed $2 \mathrm{~cm}$ below water level in the second quadrant. The detection system was programmed to terminate the trial when the animal reached the escape platform. Water temperature was maintained at $15^{\circ} \mathrm{C}-17^{\circ} \mathrm{C}$.

\subsubsection{Hidden Platform Test (Navigational Test)}

The rats were put into the pool at four compass points $(\mathrm{N}$, $\mathrm{S}, \mathrm{E}, \mathrm{W})$, the release positions around the perimeter of the maze were different for each trial, and the order was determined pseudo randomly. After the rat found the platform and remained on the platform for $30 \mathrm{sec}$, dried, given food and water, and allowed to rest for at least 10 min or until core body temperature returned to $35^{\circ} \mathrm{C}$ $37^{\circ} \mathrm{C}$. Those did not find the platform after 90 seconds counted as 90 seconds, and evasive latency was used for statistics. A test was carried each day, which lasted 4 days.

\subsubsection{Transfer Test (Probe Test)}

The platform was removed the day after hidden platform test. Rats were made to swim for 90 seconds to find the platform position with memory. The percentage of swimming distance in the platform quadrant was recorded.

\subsection{Processing of Brain Tissue}

Following water maze testing, the rats were killed by using pentobarbital followed by transcardial perfusion of the brain with cold saline, prior to being perfused with (filtered) $4 \%$ parafromaldehyde $/ 0.1 \mathrm{M}$ sodium phosphate buffer, $\mathrm{pH}$ 7.3. The brain were removed, Post-fixed in 4\% parafromaldehyde/0.1 M sodium phosphate buffer for $1 \mathrm{~h}$, cryoprotected in $0.1 \mathrm{M}$ sodium phosphate buffer containing $20 \%$ sucrose at $4^{\circ} \mathrm{C}$ for $24 \mathrm{~h}$, snap-frozen by transfer into isopentane $\left(-50^{\circ} \mathrm{C}\right)$ and stored $\left(-70^{\circ} \mathrm{C}\right)$. Each frozen brain was cut in the coronal plane $(15 \mu \mathrm{m})$ on a cryostat for histological analysis.

For electron microscopy, two rats in each group were decapitated and their brains were removed, sectioned into $2 \mathrm{~mm}$ slabs and then placed in a fixative solution of $2 \%$ sodium cacodylate-buffered glutaraldehyde, $\mathrm{pH}$ 7.4, for 6 $h$. The tissues were then rinsed in a buffered solution of saccharose, post-fixed with $1 \%$ phosphate-buffered $\mathrm{OsO}_{4}$, $\mathrm{pH}$ 7.4, dehydrated with acetone and embedded in Epon, using flat molds. The semi-thin sections, obtained with an ultramicrotome, were stained with a solution of toluidine blue and photographed under a light microscope (LM). Ultra-thin sections of selected areas were obtained using a diamond knife and stained with an alcoholic solution of uranyl acetate, followed by a solution of concentrated bismuth subnitrate. These sections were examined and photographed under an electron microscope (EM).

For histochemical staining of AChE, slices ( 2 for each rat) were washed in acetic buffer, then incubated for 30 min at $37^{\circ} \mathrm{C}$ in incubative solution (including $1 \mathrm{mM}$ Acetylthiocholine iodide); washed in $1 \%\left(\mathrm{NH}_{4}\right) \mathrm{S}, 0.1 \mathrm{~m}$ $\mathrm{NaNO}_{3}, 1 \% \mathrm{AgNO}_{3}, 0.1 \mathrm{~m} \mathrm{NaNO}_{3}$. The slices were briefly washed in PBS and then dehydrated with a graded series of ethanol solutions (from 70\% to 100\%). Slices from both staining procedures were mounted using Permount, cover-slipped, and photographed. We selected 2 visual fields from each slice, and analyzed their density using statistic method.

Activated microglia and astrocytes rapidly upregulate their expression of tissue antigens following inflammation. We visualized activated microglia using a monoclonal antibody against OX-42 (1/400 dilution, Sigma). Activated astrocytes were visualized using a polyclonal antibody against GFAP (1/200 dilution, Sigma). We used 2 slices in each group for OX-42 or GPAF immunohis- 
tochemistry reaction, selected 2 visual fields of 400 times under microscope from each slice, and analyzed their cell number and density by SPSS10.5 statistic method.

\section{Results}

Rats live normal and did not lost weight obvious after LPS injection.

\subsection{Morris Mater Testing}

\subsubsection{Hidden Platform Test}

Hidden platform showed that the average escape latency of each group rats decreased gradually with the increase of learning time. The escape latency of 6 groups is shown in Figure 1.

\subsubsection{Transfer Test}

The percentage of swimming distance in the platform quadrant of 6 groups is shown in Figure 2.

\subsection{Ultrastructural Changes in Cytoplasm}

In control group, the cytoplasm of the hippocampal neurons was filled with polyribosomes and rough endoplasmic reticulum (RER) cisternae. The Golgi complexes were numerous and prominent. In model group, on the contrary, the cytoplasm of the hipocampal neurons had an empty aspect and contained much fewer polyribosomes and RER cisternae. The Golgi complexes remained small. In PMS-treated groups and BN-treated group, the cytoplasm of the hipocampal neurons is similar to the control, there were many polyribosomes and RER cisternae, and the Golgi complexes were prominent also. The appearance of the mitochondria, lysosomes, lipofuscin pigment and cytoskeleton apparatus did not differ among all the experimental groups (Figure 3).

\subsection{Results of AChE Histochemistry}

There were a lot AChE-positive neurons and tissues in the cortex, hippocampus and basal nuclei (Figure 4). We analyzed the density of positive dye using RS IMAGE software. The results are shown in Figure 5.

\subsection{Expression of $\mathrm{OX}-42$ in Microglia and GFAP in Astrocytes}

Immunohistochemical staining for OX-42 revealed that the microglia within cerebral cortex, hippocampus and basal nuclei developed the greatest inflammatory response following acute exposure to LPS. In control group, reactive microglia were relatively fewer. In LPS group, the microglia were numerous and characterized by the contraction of their highly ramified processes, giving them a bushy morphology. P0.5 and P1 group appeared to have fewer and fewer reactive microglia than the LPS group. The number of OX-42-positive microglia was

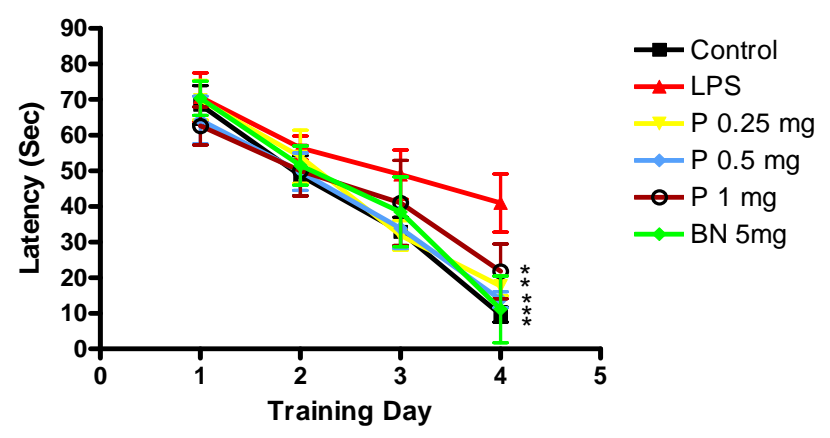

Figure 1. Escape latency of rats. $* P<0.01$, Compared with LPS group.

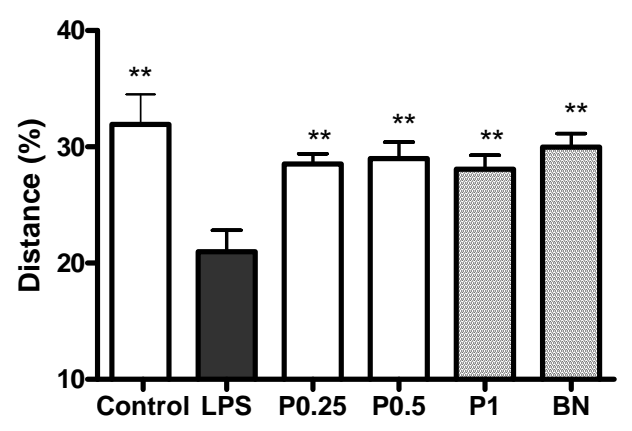

Figure 2. The percentage of swimming distance in the platform quadrant. $* * P<0.01$ vs LPS group.

shown in Figure 6; The density of OX-42-positive microglia and fibers was in Figure 7; Immunohistochemical staining for the OX-42 positive microglia was shown in Figure 8.

\subsection{Expression of GFAP in Astrocytes}

Immunohistochemical staining for GFAP revealed that the astrocytes within cerebral cortex, hippocampus and basal nuclei developed the greatest inflammatory response following acute exposure to LPS (Figure 9). The features of astrocytes in slices of 4 groups' rats were the same as miroglia. The number and density of GFAPpositive astrocytes are shown in Figures $\mathbf{1 0}$ and $\mathbf{1 1 .}$

\section{Discussion}

The underlying mechanism of HAD involve neuroinflammation induced by invading of HIV-1, and neuroinflammation gives rise to neurons impairment, including cholinergic neuron [8]. Inhibiting neuroinflammation and ameliorating cholinergic nerve function may be the two therapy of HAD. It was reported that chronic infusions of LPS impaired spatial memory, destroyed cholinergic cells and activated astrocytes and microglia, and induced neuroinflammation [9]. In this report, a neuroinflammatory murine model was used to explore the relationship between cognitive function, physiology, and neuropa 

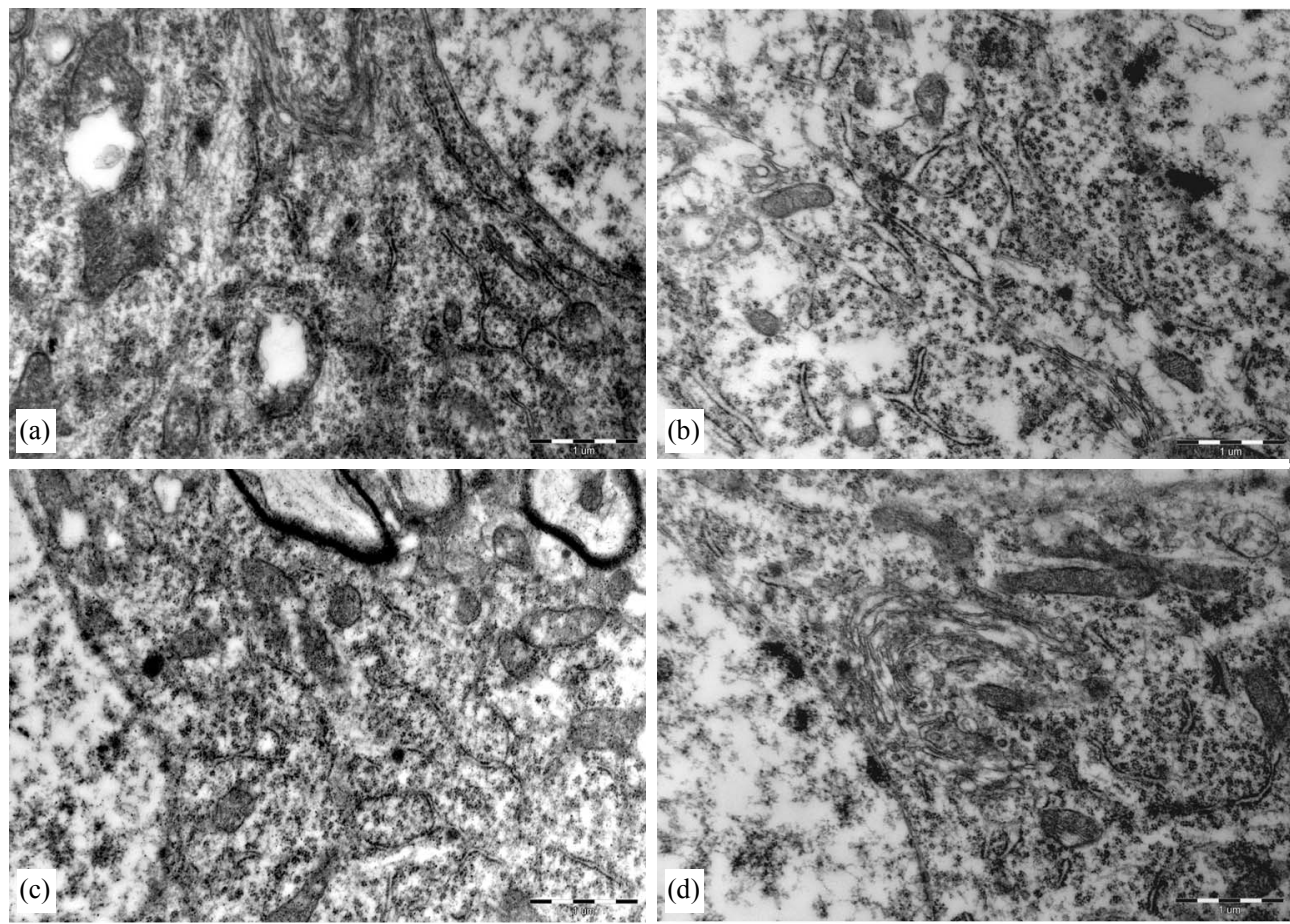

Figure 3. Electron micrograph of a hippocampal neurons. (a) Control group, there were much polyribosomes and rough endoplasmic reticulum (RER) cisternae; (b) LPS group, the hipocampal neurons had an empty aspect and contained much fewer polyribosomes and RER cisternae; (c) (BN group) and (d) (P0.5 group), there were more polyribosomes and RER cisternae than that of LPS group.
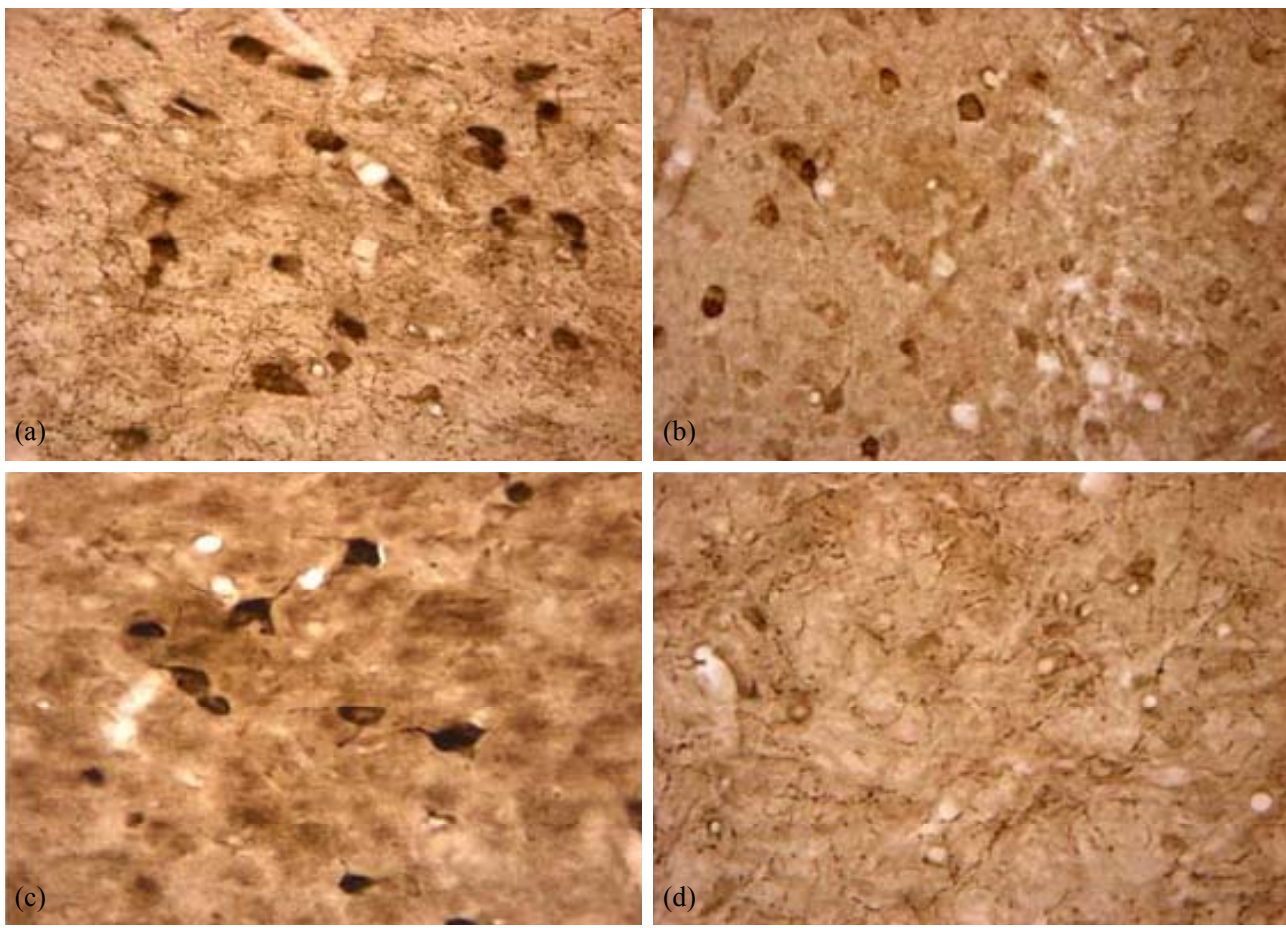

Figure 4. AChE positive neurons and tissues in the basal nuclei $(\times 400)$. (a) Control group, there were many AChE positive neurons and tissues and they were dyed dark brown; (b) LPS group, The AChE positive neurons were dyed beige; (c) BN group, there were also many AChE positive neurons and fibers, and there were dyed dark brown; (d) P0.5, The positive dyes were much beige. 


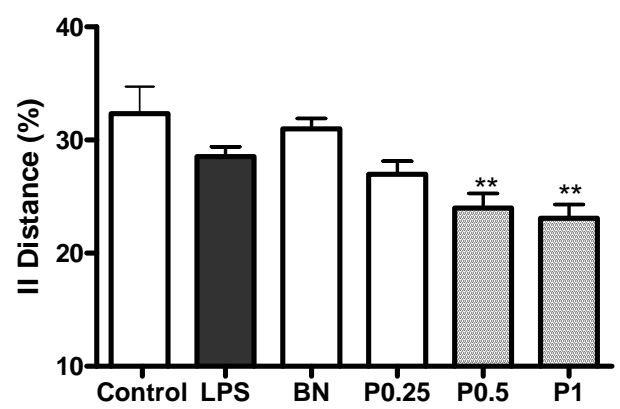

Figure 5. The density of AChE positive neurons and tissues in the cortex, hippocampus and basal nuclei of 6 groups' rats. $* * P<0.01$ vs control group.

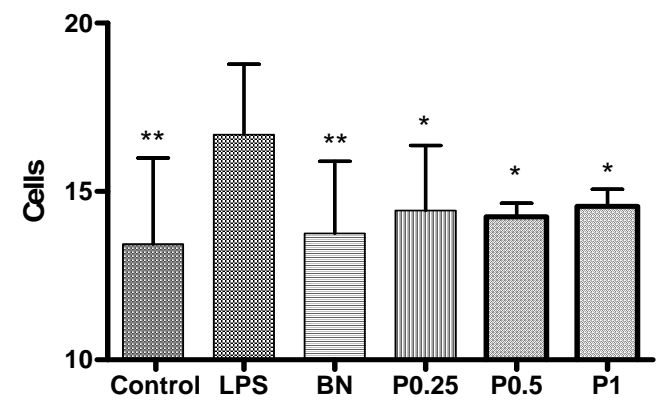

Figure 6. The number of $\mathrm{OX}-42$-positive microglia in the cortex, hippocampus and basal nuclei. $* P<0.05$, ${ }^{* *} P<0.01$ vs LPS group.

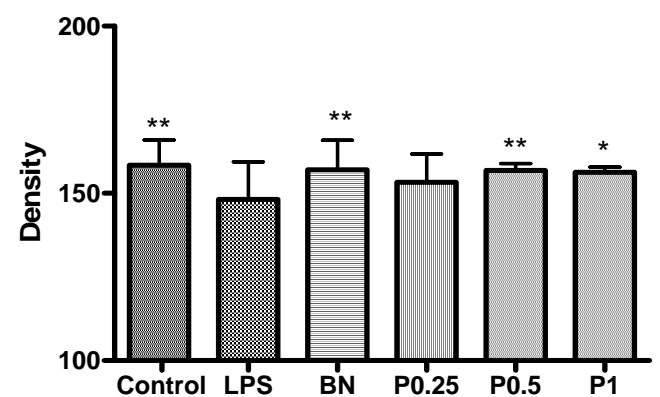

Figure 7. The density of $\mathrm{OX}-42$-positive microglia and fibers in the cortex, hippocampus and basal nuclei. $* P<0.05$ vs LPS group.

thology after injection of LPS into the forth ventricle [10]. Moreover, we here report that peripheral administration of the new compound PMS777-a PAFR antagonism and AchE inhibitor could significantly improve spatial memory, attenuate the cytotoxic consequences of chronic neuroinflammation upon cerebral cortex, hippocampus and basal nuclei. PMS777 had previously been shown to provide neuroprotectoin against oxidative injury induced in human neuroblastoma SK-N-SH cells and oxidative stress and mitochondrial impairment in human HepG2 cells [11-13].

The central inflammatory response produced by infu- sion of LPS into the 4th ventricle is associated with an increase in the number of activated astrocytes and reactive microglia distributed throughout the brain. The greatest inflammatory response occurred within the hippocampus, basal nuclei and cerebral cortex. The inflammation was also associated with an increased of IL- $1 \alpha$, IL- $1 \beta$, PAF and prostaglandin levels, particularly within the basal forebrain and hippocampus [14]. On the other hand, increased PAF production has been implicated in HAD. Increased PAF can directly and indirectly increase glutamate in the extracellular space [15], leading to the stimulation of glutamate and NMDA receptors, which can result in neuronal death. In deed, cholinergic neurons of basal nuclei and hippocampus receive a dense glutamatergic projection from the pedunculopontine tegmentum, thus they are potentially vulnerable to excess stimulation of glutamatergic NMDA receptors [16].

In the present study, the neurobiological consequences of the acute neuroinflammation are a significant deficit in spatial memory. The behavioral impairment may be due to the degeneration of hippocampal pyramidal neurons, cerebral cortex neurons and basal nuclei neurons. The cytotoxicity of LPS can be completely reversed by coadministration of PMS777. These results indicated that the cytotoxic effects of acute LPS infusion may be due to PAF releasing and decrease of acetylcholine.

The electron microscopic study revealed numerous changes in the cellular components involved in protein synthesis within the hippocampal neurons of LPS-treated rats. These changes probably underlie the significant learning and memory impairments. The cytoplasm of these cells contained few polyribosomes, few RER cisternae and a small Golgi apparatus, all these suggested that function of protein synthesis was impaired or reduced. Both these ultrastructural changes inside neurins cytoplasm may represent an attempt to compensate for impaired protein synthesis. PMS777 had been shown to enhance the number of polyribosomesand RER cisternae and Golgi apparatus size of hippocampal neurons. It indicated that PMS777 could attenuate the dysfunction of protein synthesis.

These studies showed a significant increase in the number of activated microglia and astrocytes within brains of LPS-treated rats. Our data were consistent with the hypothesis that long-term inflammation within the brain may lead to degenerative changes, particularly within the hippocampus [17]. These degenerative changes might underlie the age-associated deficit in spatial memory ability that has been widely documented in rats. The greatly enhanced inflammatory response observed within the cerebral cortex and basal nuclei in the studies might explain why these regions are associated with such profound pathological changes in the brains of patients with 

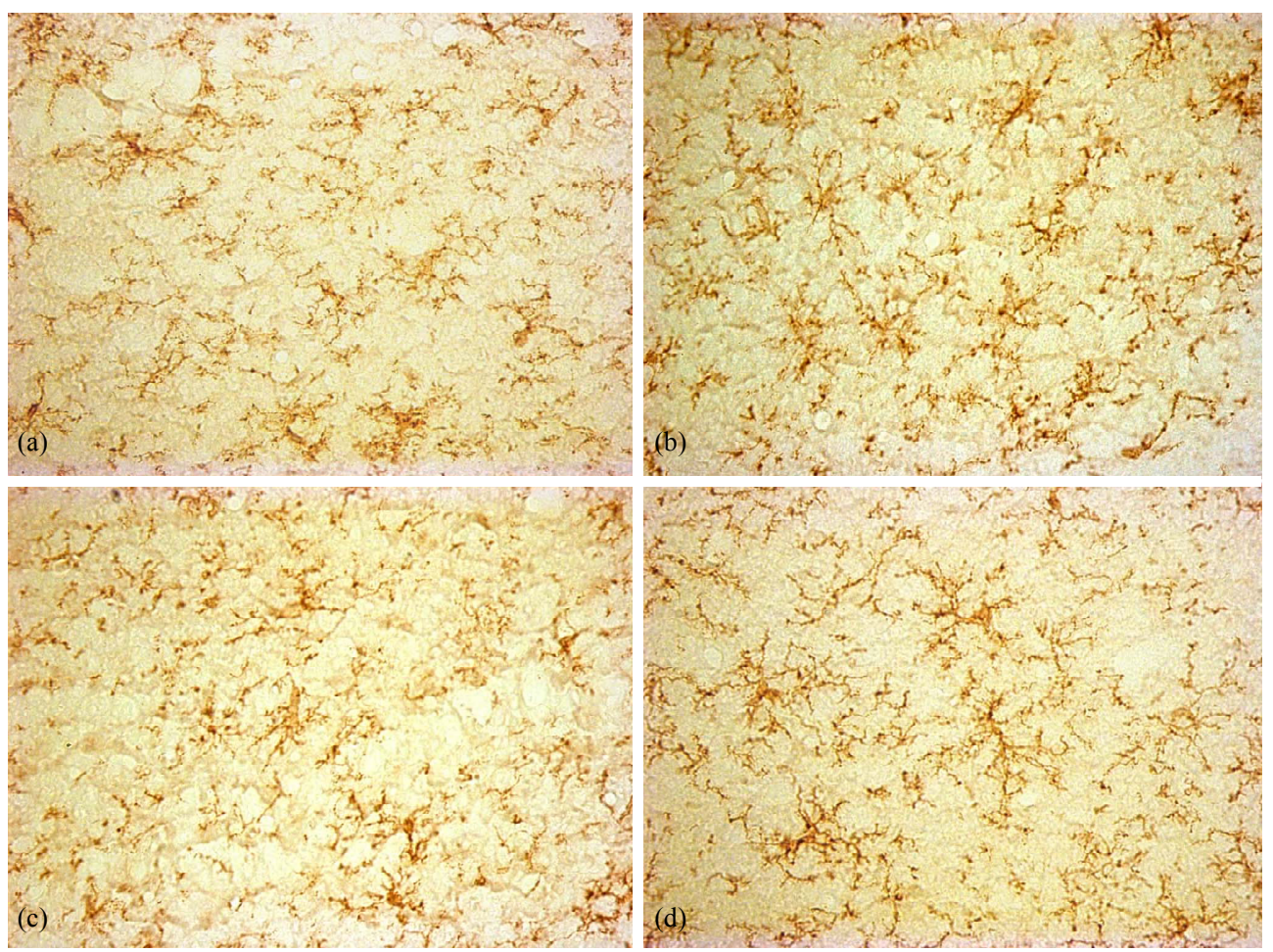

Figure 8. Immunohistochemical staining for the OX-42 antibody $(\times 400)$. (a) Control group, A few OX-42-positive microglia were seen in hippocampus; (b) LPS group, Activated microglia distributed throughout the hippocampus; (c) and (d) BN and P0.5, the activated microglia were less than LPS group.
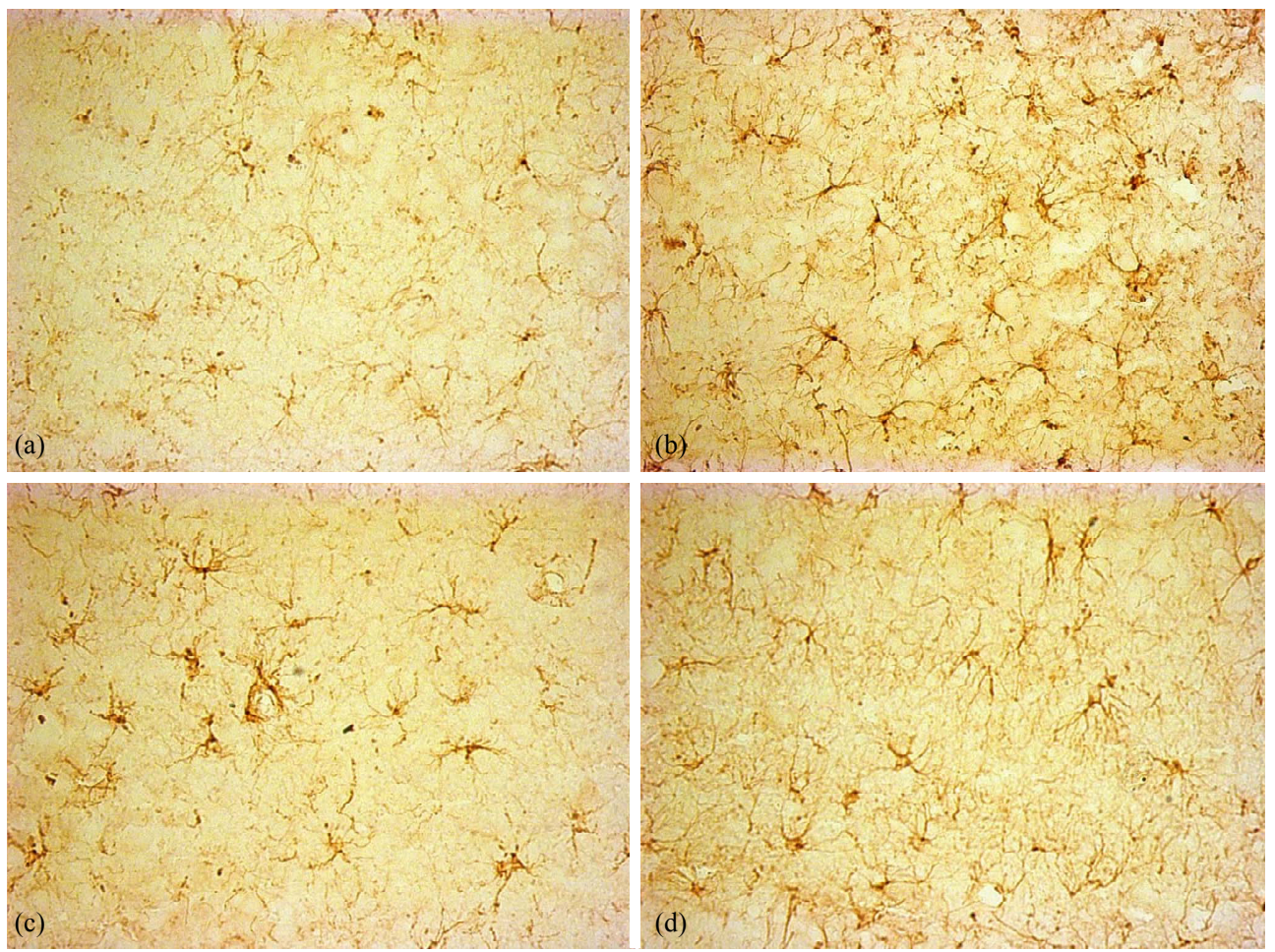

Figure 9. Immunohistochemical staining for the GFAP antibody. (×400). (a) Control group, few GFAP-positive astrocytes were seen in hippocampus; (b) LPS group, activated astrocytes distributed throughout the hippocampus; (c) and (d): BN and P0.5, the activated microglia were less than LPS group. 


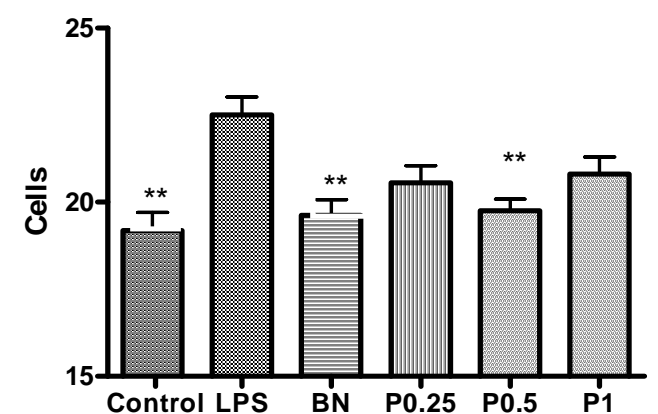

Figure 10. The number of GFAP-positive astrocytes in the cortex, hippocampus and basal nuclei of 6 groups. $* P<0.05$ vs LPS.

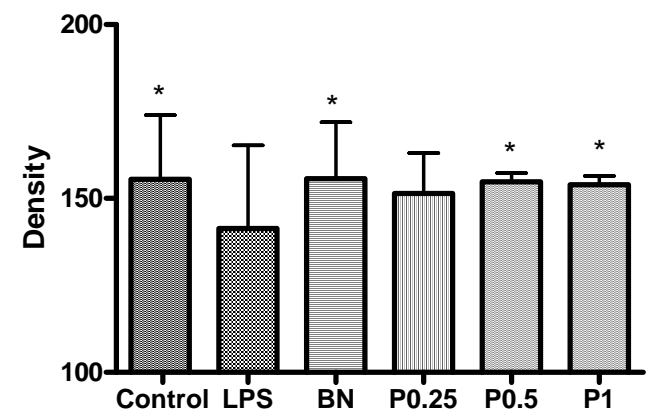

Figure 11. The density of GFAP-positive neurons and fibers in the cortex, hippocampus and basal nuclei of 6 groups. $* P$ $<0.05$ vs LPS group.

HAD. Administration of PMS777 has been shown to decrease the number of activated microglia and astrocytes induced by LPS.

There is little effective therapy for HAD. Clinical drug trials in patients with Alzheimer's disease have focused on drugs that augment levels of $\mathrm{ACh}$ in the brain to compensate for the loss of cholinergic function, which is the main source of recognition and memory impairment. Thus suggest increasing levels of ACh can also improve recognition and memory in HAD patients. These drugs have included ACh precursors, muscarinic agonists, nicotinic agonists, and acetylcholinesterase inhibitors [18]. The most highly developed and successful approaches to date have employed acetylcholinestrase inhibition. Non-steroidat anti-inflammatory drugs are widely used in neurodegeneration disease such $\mathrm{AD}$ or frontotemporal dementia, which provide therapeutic benefit [19]. So several studies indicated that bifunctional or multifunctional compounds might provide greater efficacy in neuroprotection than single-target compounds [20]. Thus therapeutic strategics focusing on two or more targets might provide greater benefit in neurodegeneration diseases treatment. Moreover, drugs with multifunctional actions might be more proficient compared with multiple drugs combinations because they simplify ad- ministration and obviate the different degrees of bioavailability and pharmacokinetics of multiple single-target drugs. PMS777-a new acetylcholinesterase inhibitor and PAFR antagonist improve spatial memory, ameliorate cholinergic function and inhibit neuroinflammation process in LPS-treated rat, our animal model with some feature of HIV-associated dementia or Alzheimer's disease. PMS777 might be used in clinical trials of AD or HAD.

\section{REFERENCES}

[1] H. Dou, J. Morehead, J. Bradley, et al., "Neuropathologic and Neuroinflammatory Activities of HIV-1-Infected Human Astrocytes in Murine Brain," Glia, Vol. 54, No. 2, 2006, pp. 81-93. doi:10.1002/glia.20358

[2] H. Dou, J. D. Kingsley, R. L. Mosley, et al., "Neuroprotective Strategies for HIV-1 Associated Dementia," Neurotoxicity Research, Vol. 6, No. 7-8, 2004, pp. 503-521. doi:10.1007/BF03033447

[3] J. J. Hoozemans, R. Veerhuis, I. Janssen, et al., "Interleukin-1beta Induced Cyclooxygenase 2 Expression and Prostaglandin E2 Secretion by Human Neuroblastoma Cells: Implications for Alzheimer's Disease," Experimental Gerontology, Vol. 36, No. 3, 2001, pp. 559-570. doi:10.1016/S0531-5565(00)00226-6

[4] R. M. Bluthe, R. Dantzer and K. W. Delley, "Effects of Interleukin-1 Receptor Antagonist on the Behavioral Effects of Lipopolysaccharide in Rat," Brain Research, Vol. 573, No. 2, 1992, pp. 318-320. doi:10.1016/0006-8993(92)90779-9

[5] N. Quan, S. K. Sundar and J. M. Weiss, "Induction of Interleukin-1 in Various Brain Regions after Peripheral and Central Injections of Lipopolysaccharide," Journal of Neuroimmunology, Vol. 49, No. 1-2, 1994, pp. 125-134. doi:10.1016/0165-5728(94)90188-0

[6] C. Sherwin and R. Fern, "Acute LipopolysaccharideMediated Injury in Neonatal White Matter Glia: Role of TNF-alpha, IL-1beta, and Calcium," Journal of Immunology, Vol. 175, No. 1, 2005, pp. 155-161.

[7] W. E. Zink, E. Anderson, J. Boyle, et al., "Impaired Spatial Cognition and Synaptic Potentiation in a Murine Model of Human Immunodeficiency Virus Type 1 Encephalitis," Journal of Neuroscience, Vol. 22, No. 6, 2002, pp. 2096-2105.

[8] Y. Huang, N. Erdmann, J. Zhao, et al., "The Signaling and Apoptotic Effects of TNF-Related Apoptosis-Inducing Ligand in HIV-1 Associated Dementia," Neurotoxicity Research, Vol. 8, No. 1-2, 2005, pp. 135-148. doi:10.1007/BF03033825

[9] H. W. Beatrice, G. V. Maria and L. W. Gary, "Behavioral and Ultrastructural Changes Induced by Chronic Beuroinflammation in Young Rats," Brain Research, Vol. 859, 2000, pp. 157-166. doi:10.1016/S0006-8993(00)01999-5

[10] B. Hauss-Wegizyniak, L. Lukovic, M. Bigaud, et al., "Brain Inflammatory Response Induced by Intracere- 
broventricular Infusion of Lipopolysaccharide: An Immunohistochemical Study," Brain Research, Vol. 794, No. 2, 1998, pp. 211-224. doi:10.1016/S0006-8993(98)00227-3

[11] M. F. Ezoulin, C. Z. Dong, Z. Liu, J. Li, H. Z. Chen, et al., "Study of PMS777, a New Type of Acetylcholinesterase Inhibitor, in Human HepG2 Cells, Comparison with Tacrine and Galanthamine on Oxidative Stress and Mitochondrial Impairment," Toxicology in Vitro, Vol. 20, No. 6, 2006, pp. 824-831. doi:10.1016/j.tiv.2006.01.002

[12] J. Li, H. Huang, J. M. Miezan Ezoulin, et al., "Pharmacological Profile of PMS777, a New AChE Inhibitor with PAF Antagonistic Activity," International Journal of Neuropsychopharmacology, Vol. 10, No. 1, 2007, pp. 2129. doi:10.1017/S1461145705006425

[13] M. J. Ezoulin, J. Li, G. Wu, et al., "Differential Effect of PMS777, a New Type of Acetylcholinesterase Inhibitor, and Galanthamine on Oxidative Injury Induced in Human Neuroblastoma SK-N-SH Cells," Neuroscience Letters, Vol. 389, No. 2, 2005, pp. 61-65. doi:10.1016/j.neulet.2005.07.026

[14] R. L. Richardson, E. M. Kim, T. Gardiner and E. O'Hare, "Chronic Intracerebroventricular Infusion of Lipopolysaccharide: Effects of Ibuprofen Treatment and Behavioural and Histopathological Correlates," Behavioural Pharmacology, Vol. 16, No. 7, 2005, pp. 531-541. doi:10.1097/01.fbp.0000179278.03868.96

[15] Y. Persidsky, J. Limonges, J. Rasmussen, et al., "Reduction in Glial Immunity and Neuropathology by a PAF Antagonist and an MMP and TNFalpha Inhibitor in SCID
Mice with HIV-1 Encephalitis," Journal of Neuroimmunology, Vol. 114, No. 1-2, 2001, pp. 57-68. doi:10.1016/S0165-5728(00)00454-9

[16] T. Pung, B. Klein, D. Blodgett, et al., "Examination of Concurrent Exposure to Repeated Stress and Chlorpyrifos on Cholinergic, Glutamatergic, and Monoamine Neurotransmitter Systems in Rat Forebrain Regions," International Journal of Toxicology, Vol. 25, No. 1, 2006, pp. 65-80. doi:10.1080/10915810500527119

[17] M. Hartlage-Rubsamen, R. Lemke and R. Schliebs, "Interleukin-1beta, Inducible Nitric Oxide Synthase, and Nuclear Factor-KappaB Are Induced in Morphologically Distinct Microglia after Rat Hippocampal Lipopolysaccharide/Interferon-Gamma Injection," Journal of Neuroscience Research, Vol. 57, No. 3, 1999, pp. 388-398. doi:10.1002/(SICI)1097-4547(19990801)57:3<388::AIDJNR11>3.0.CO;2-2

[18] C. G. Ballard, "Advances in the Treatment of Alzheimer's Disease: Benefits of Dual Cholinesterase Inhibition," European Neurology, Vol. 47, No. 1, 2002, pp. 64 70. doi:10.1159/000047952

[19] S. H. Wang, J. Li and M. Y. Geng, "Inflammatory Pathology and the Anti-Inflammation of Saccharide Substances in Alzheimer's Disease," Sheng Li Ke Xue Jin Zhan, Vol. 36, No. 1, 2005, pp. 67-70.

[20] M. B. H. Youdim and J. J. Buccafusco, "Multi-Functional Drugs for Various CNS Targets in the Treatment of Neurodegenerative Disorders," Trends in Pharmacology Science, Vol. 26, 2005, pp. 27-35. doi:10.1016/j.tips.2004.11.007 